Article

\title{
Preparation of Ion-Exchanged TEMPO-Oxidized Celluloses as Flame Retardant Products
}

\author{
Cunzhen Geng ${ }^{1,2,3, *}$, Zhihui Zhao ${ }^{1,2,3}$, Zhixin Xue ${ }^{1,2,3}$, Peilong $\mathrm{Xu}^{1, *}$ and Yanzhi Xia ${ }^{1,2,3, *}$ \\ 1 State Key Laboratory of Bio-fibers and Eco-textiles, Qingdao University, Qingdao 266071, China \\ 2 Institute of Marine Biobased Materials, Qingdao University, Qingdao 266071, China; zzh@qdu.edu.cn (Z.Z.); \\ xuezhixin@qdu.edu.cn (Z.X.) \\ 3 Co-Innovation Center for Marine Biomass Fibers, Materials and Textiles of Shandong Province, \\ Qingdao University, Qingdao 266071, China \\ * Correspondence: qdugcz@qdu.edu.cn (C.G.); xpl@qdu.edu.cn (P.X.); xiayz@qdu.edu.cn (Y.X.); \\ Tel.: +86-532-85952779 (C.G.); +86-532-85952779 (P.X.); +86-532-85953069 (Y.X.)
}

Received: 22 April 2019; Accepted: 17 May 2019; Published: 21 May 2019

\begin{abstract}
Cellulose, as one of the most abundant natural biopolymers, has been widely used in textile industry. However, owing to its drawbacks of flammability and ignitability, the large-scale commercial application of neat cellulose is limited. This study investigated some TEMPO-oxidized cellulose (TOC) which was prepared by selective TEMPO-mediated oxidation and ion exchange. The prepared TOC was characterized by Fourier transform infrared (FT-IR) spectroscopy and solid-state ${ }^{13} \mathrm{C}$-nuclear magnetic resonance $\left({ }^{13} \mathrm{C}-\mathrm{NMR}\right)$ spectroscopy. The thermal stability and combustion performance of TOC were investigated by thermogravimetry analysis (TG), microscale combustion calorimetry (MCC) and limiting oxygen index (LOI). The results demonstrated that the thermal stability of TOC was less than that of the pristine material cellulose, but the peak of heat release rate (pHHR) and the total heat release (THR) of all TOC were significantly reduced. Additionally, the LOI values of all TOC products were much higher $25 \%$. In summary, the above results indicated that the modified cellulose with carboxyl groups and metal ions by selective oxidation and ion exchange endows efficient flame retardancy.
\end{abstract}

Keywords: cellulose; TEMPO-oxidized cellulose (TOC); thermal stabilization; microscale combustion calorimeter (MCC); flame retardancy

\section{Introduction}

Cellulose, the most abundant renewable polysaccharide on Earth, has safe, biocompatible, hydrophilic, and biodegradable natures, and is one of the best candidates for textile and other functional materials. Cellulose has broad potential in the design of advanced polymeric materials because of its linear $(1,4)-\beta$-glucan structure with three reactive hydroxyl groups per anhydroglucopyranose unit [1]. It is well-known that the cellulose is also one of the most flammable materials (limiting oxygen index (LOI) 18.4\%) and can be ignited easily [2,3]. However, it is of primary importance for public safety to find ways to render this material less flammable and, of course, in a most economical and environment friendly manner. Consequently, to endow cotton fabric with excellent flame retardancy, flame-retardant modification is necessary.

In the last years, many flame retardants which contained halogen, phosphorous, nitrogen, sulfur, silicon, boron, and aluminum have been studied to endow cellulose with highly efficient flame-retardant property [4-7]. Although these flame retardants can effectively enhance the flame-retardancy of textile, some have been shown to be hazardous for human health and the ecological environment. Moreover, some of these flame retardants are easily washed out and not durable. Therefore, it is necessary to 
develop other methods to improve the flame retardancy of cellulose, for instance, some stable groups (anionic phosphate groups) are introduced to cellulose macromolecular chain through chemical bond to improve the flame-retardant property of cellulose [8].

Alginic acid, a kind of linear polysaccharide, is derived from brown seaweed. It is a co-polymer composed of $\mathrm{b}$-1, 4-D-mannuronate $(\mathrm{M})$ and a -1,4-L-guluronate $(\mathrm{G})$ repeating monomeric units with the chemical formula $\left(\mathrm{C}_{6} \mathrm{H}_{8} \mathrm{O}_{6}\right)_{n}$. In our laboratory, we already found that alginate fibers have a potential and intrinsically flame-retardant property without the addition of any other flame retardants. Kong, Q.S. et al. found that calcium alginate fibers, prepared by wet spinning of sodium alginate into a coagulating bath containing calcium chloride, are inherently flame retardant with a LOI value of $34 \%$ [9]. Zhang, J.J. et al. did further research, which demonstrated that calcium alginate fiber was intrinsically flame retardant with LOI value of $48 \%$ and proposed a condensed phase mechanism for the calcium alginate fiber flame retardancy effect [10]. Alginate fibers usually contain some $\mathrm{Na}$, $\mathrm{Ca}$, or other salts, and the presence of these ions will increase their flame-retardant property. More importantly, the presence of a carboxylate/carboxylic acid group will behave as a Lewis acid when alginate is heated, thereby producing char and increasing the value of LOI. The most typical method to introduce carboxyl groups into cellulose is carboxymethylation with monochloroacetic acid under alkaline conditions generally containing i-propanol, and large amounts of carboxymethyl cellulose (CMC) have been produced at industrial level.

By comparing the two chemical structures of alginate and cellulose (Figure 1), a distinct difference is that a primary hydroxyl group located in C6 position of cellulose and a carboxylic acid group in corresponding position of alginate.

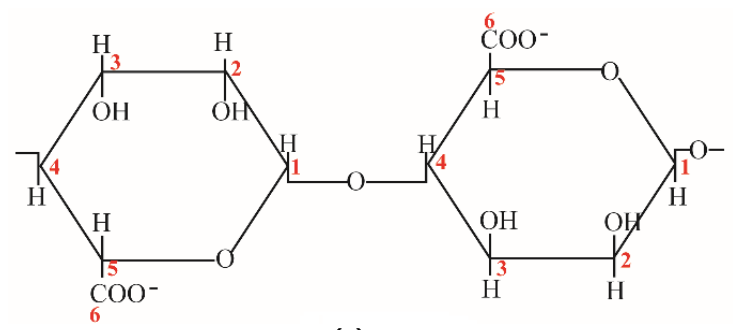

(a)

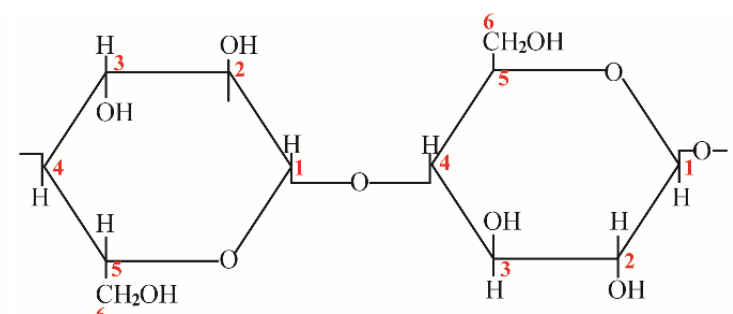

(b)

Figure 1. Chemical structure of alginate (a) and cellulose (b).

Based on this information, we tried to find a method to realize the transformation from a primary alcohol group to a carboxyl group in C6 position. A 2,2,6,6- tetramethylpiperidine-1-oxyl radical (TEMPO)-mediated oxidation is a system of the position-selective chemical modifications of primary hydroxyl groups, undertaken by reaction with TEMPO, sodium hypochlorite, and sodium bromide in water. It was an effective method to convert the primary hydroxyl groups of polysaccharides, such as sulfite pulp, cotton, bacterial cellulose, etc., to carboxyl groups with high selectivity [11-14].

In the present paper, we attempted to selectively oxidize the wood pulp whose chemical structure is the same as cellulose by the TEMPO-mediated oxidation system in water to obtain TEMPO-oxidized cellulose (TOC). Furthermore, the burning behavior and thermal stability of TOC were further investigated by MCC tests and TG analysis, respectively. To the best of our knowledge, this is the first reported flame-retardant behavior observed in cellulose by modifying the C6 group in its molecular structure.

\section{Results and Discussion}

\subsection{FT-IR Spectroscopy Analysis}

FT-IR spectra of the cellulose and cellulose-COONa are shown in Figure 2. The wood pulp has almost no absorption band from 1400 to $1700 \mathrm{~cm}^{-1}$, but two strong absorption bands at 1608 and $1426 \mathrm{~cm}^{-1}$ derived from the carbonyl groups present in response to the TEMPO-mediated oxidation of 
wood pulp, which are used for quantification purposes representing the appearance of $v_{a s}(C=O)$ and $v_{\mathrm{s}}(\mathrm{C}-\mathrm{O})$, respectively. In addition, a slight reduction of the bands related to hydrogen bonds $v(\mathrm{OH})$ at $3377 \mathrm{~cm}^{-1}$ and $v(\mathrm{CH})$ at $2902 \mathrm{~cm}^{-1}$ were observed. The results are consistent with other reports [14,15]. From these results, it can be concluded that hydroxyl groups at the C6 position of cellulose molecules are converted to sodium carboxylate and the wood pulp can be selectively oxidized in C6 position of cellulose molecules with TEMPO-mediated oxidation system.

Additionally, all the FT-IR spectra of TOC are presented in Figure 3. The peak at $1608 \mathrm{~cm}^{-1}$ was attributed to the antisymmetric stretching vibration peak of carboxylic group (-COO- )and carbonyl $(\mathrm{C}=\mathrm{O})$, and the peaks at $1426 \mathrm{~cm}^{-1}, 3377 \mathrm{~cm}^{-1}$, and $2902 \mathrm{~cm}^{-1}$ were assigned to $v(\mathrm{C}-\mathrm{O}), v(\mathrm{OH})$, and $v\left(\mathrm{CH}_{2}\right)$, respectively. All the FT-IR spectra bands of five TOC samples were almost the same. It indicates that the introduction of various metal ions into cellulose-COONa through ion exchange had no effect on the position and strength of these specific peaks.

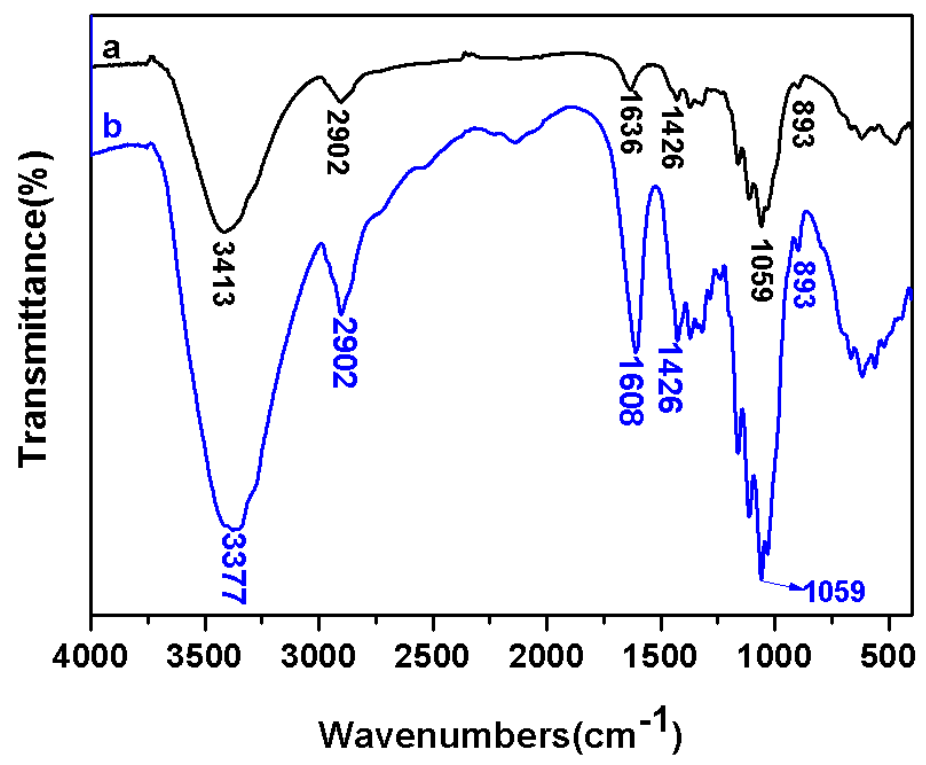

Figure 2. FT-IR spectra of the original cellulose (a) and cellulose-COONa (b).

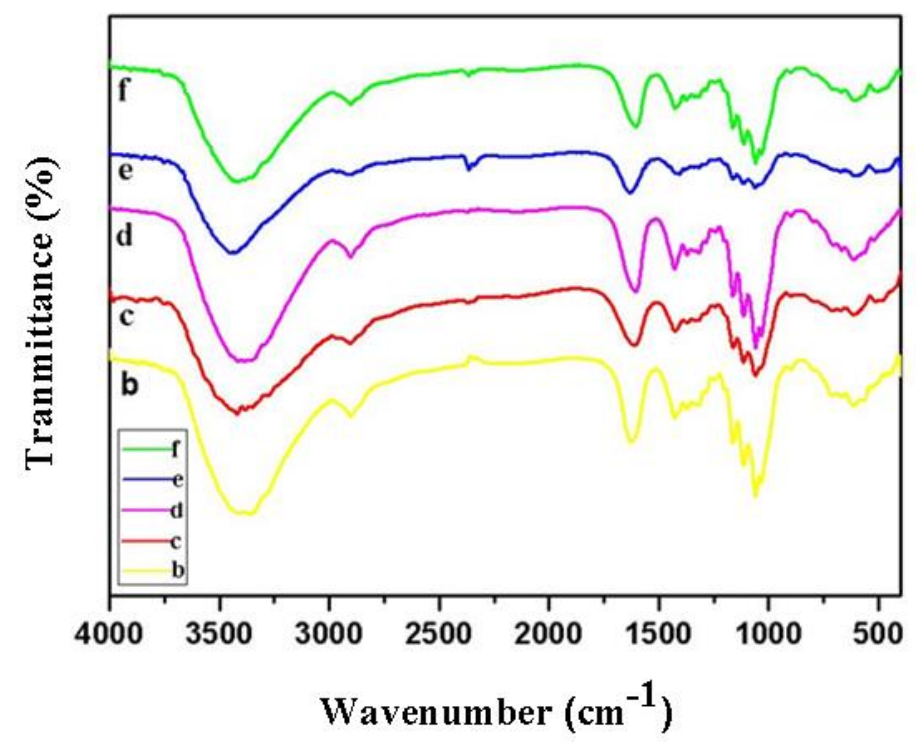

Figure 3. FT-IR spectra of cellulose-COOZn (b), cellulose-COOMg (c), cellulose-COOCa (d), cellulose-COONa (e), and cellulose-COOBa (f). 


\section{2. ${ }^{13}$ C-NMR Spectroscopy Analysis}

Solid-state ${ }^{13} \mathrm{C}-\mathrm{NMR}$ spectra of original cellulose and TEMPO-oxidized cellulose sample are shown in Figure 4. For the original cellulose, the typical signals of cellulose appear at $107 \mathrm{ppm}(\mathrm{C} 1)$, 90 and 85 ppm (C4), 76 and 73 ppm (C2, C3, and C5), and 66.6 and 64.4 ppm (C6). A new peak at $176 \mathrm{ppm}$ (see Figure $4 \mathrm{~b}$ ) is ascribed to the sodium carboxylate carbons in the oxidized cellulose, when compared with the original cellulose (see Figure 4a). These results are consistent with the previous relevant reports $[16,17]$.

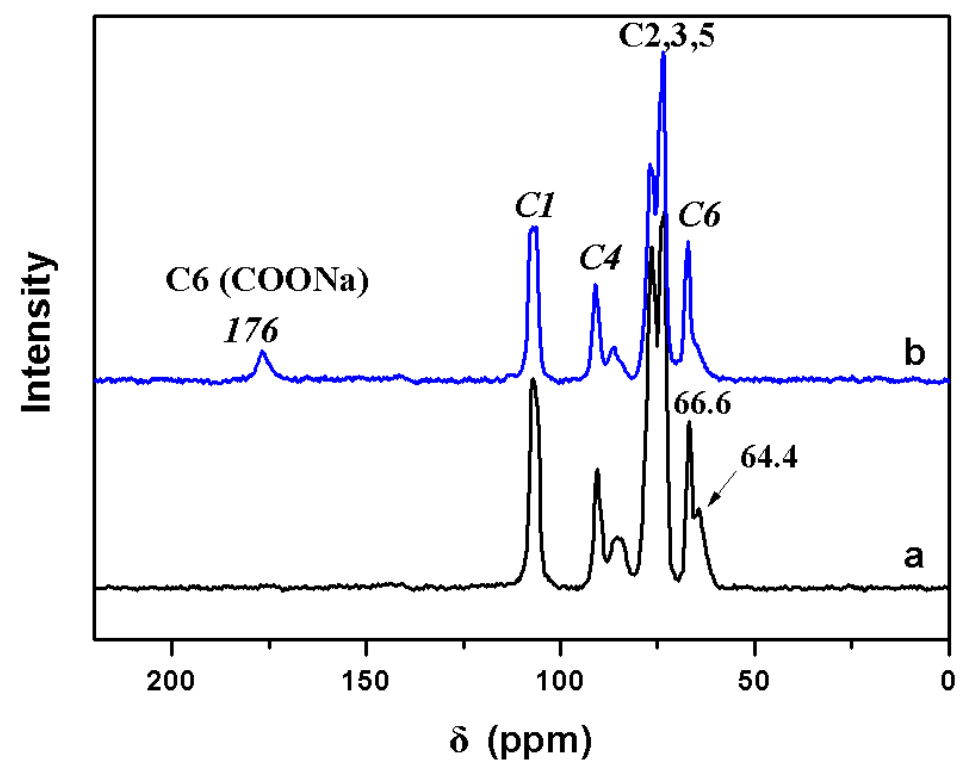

Figure 4. Solid-state ${ }^{13} \mathrm{C}-\mathrm{NMR}$ spectra of the original cellulose (a) and cell-COONa (b).

From Figure 4, we find that cellulose-COONa had nearly no influences on the chemical shift and the pattern of $\mathrm{C} 1$ or $\mathrm{C} 4$ in solid-state ${ }^{13} \mathrm{C}-\mathrm{NMR}$ spectra. However, the oxidized cellulose has large resonance peaks due to the original C6 primary alcohol groups of the glucose at about $67.3 \mathrm{ppm}$. Meanwhile, the signal at $64.4 \mathrm{ppm}$ for noncrystalline $\mathrm{C} 6$ carbons sharply decreases and disappears when it is compared with the original cellulose, which indicates a selective oxidation of the primary $\mathrm{OH}$-units [18]. Thus, it is possible to conclude that selective oxidation occurred at the C6 primary alcohol groups of wood pulp in TEMPO-NaBr-NaClO system.

\subsection{TG Analysis}

The thermal stability of cellulose and TOC were studied by thermal weight loss under nitrogen atmosphere. The TG and DTG curves were showed in Figures 5 and 6, respectively. The TG and DTG data of the cellulose and TOC were listed in Table 1 in detail.

As can be seen from Figures 5 and 6 , and Table 1, the initial decomposition temperature $\left(T_{0.1}\right)$, maximum mass loss temperature ( $\mathrm{T}_{\max }$ ) and peak temperatures of derivative TG (DTG peak) of all TOC are lower than that of the pristine cellulose. It indicates that thermal stability of the original cellulose was significantly reduced after modification. It is possibly because of the increase of carboxyl groups and the addition of metal ions due to the decrease in crystallinity of TOC during the oxidation and ion-exchange [19].

However, the maximum weight loss rate (MMLR) of TOC was significantly lower than that of cellulose from Table 1. Some possible reasons can be speculated in that various TOC which contain more carboxyl groups will release a large amount of nonflammable gas $\left(\mathrm{CO}_{2}\right)$ and dilute the flammable gas during degradation at a lower temperature. Additionally, it is more easily to generate carbon residue at a relatively low temperature [20]. 
We also found that the amount of residual combustion residue of TOC was significantly increased from Table 1. Generally, the formation of combustion residue will block oxygen ingress, slow down heat transfer, and prevent flame spread, and the more residual combustion residue, the more obvious are these effects during the process of polymer degradation.

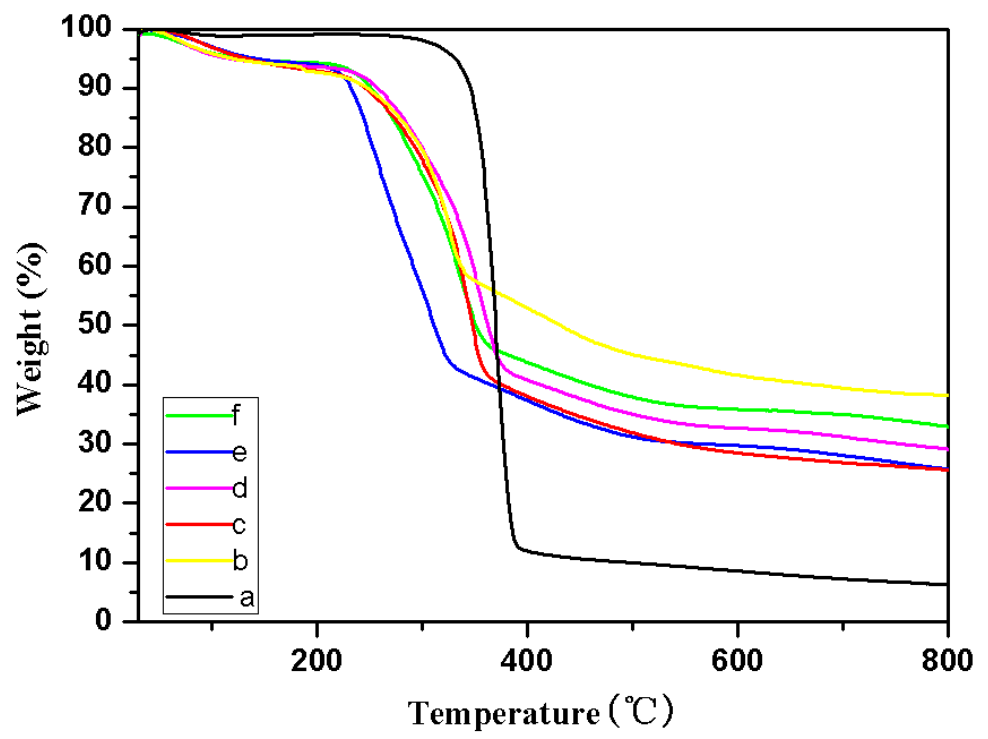

Figure 5. TG curves of cellulose (a), cellulose-COOZn (b), cellulose-COOMg (c), cellulose-COOCa (d), cellulose-COONa (e), and cellulose-COOBa (f) under $\mathrm{N}_{2}$.

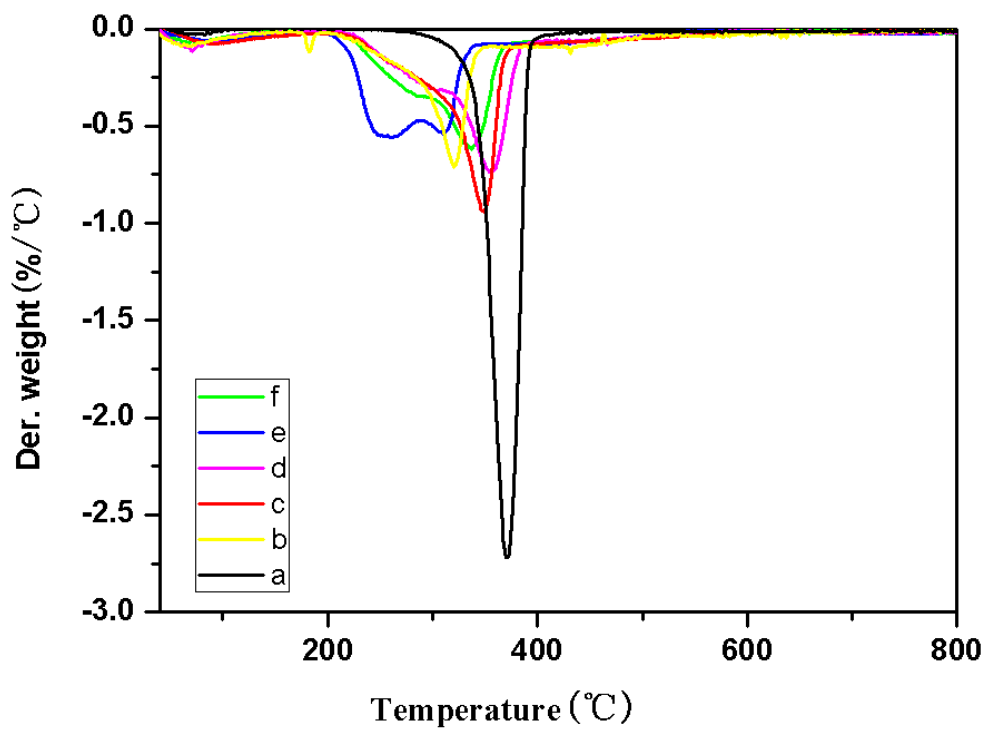

Figure 6. DTG curves of cellulose (a), cellulose-COOZn (b), cellulose-COOMg (c), cellulose-COOCa (d), cellulose-COONa (e), and cellulose-COOBa (f) under $\mathrm{N}_{2}$.

Table 1. The TG and DTG data of cellulose and TEMPO-oxidized cellulose (TOC).

\begin{tabular}{cccccc}
\hline Sample & $\mathbf{T}_{\mathbf{0 . 1}}\left({ }^{\circ} \mathbf{C}\right)$ & DTG $_{\text {peak }}\left({ }^{\circ} \mathbf{C}\right)$ & $\mathbf{T}_{\text {max }}\left({ }^{\circ} \mathbf{C}\right)$ & Combustion Residue $\left(\mathbf{8 0 0}{ }^{\circ} \mathbf{C}, \mathbf{w t} \%\right)$ & MMLR $\left(\% /{ }^{\circ} \mathbf{C}\right)$ \\
\hline cellulose & 300 & 367 & 367 & 6.20 & 2.82 \\
cellulose-COOZn & 231 & 319 & 319 & 33.05 & 0.73 \\
cellulose-COOMg & 225 & 339 & 339 & 25.64 & 1.14 \\
cellulose-COOCa & 240 & 349 & 349 & 29.08 & 0.77 \\
cellulose-COONa & 229 & $260 ; 307$ & 260 & 25.70 & 0.56 \\
cellulose-COOBa & 243 & 334 & 334 & 32.92 & 0.61 \\
\hline
\end{tabular}

$\mathrm{T}_{0.1}$ : Temperature in weight loss of $10 \%$; $\mathrm{DTG}_{\text {peak }}$ : Peak temperatures of derivative $\mathrm{TG} ; \mathrm{T}_{\max }\left({ }^{\circ} \mathrm{C}\right)$ : Maximum mass loss temperature; MMLR: Maximum mass loss rate. 


\subsection{Analysis}

The flame retardancy of cellulose and TOC were tested and characterized using microscale combustion calorimetry (MCC). MCC analysis is a new, rapid, and convenient method that has become an efficient tool for characterizing newly synthesized flame-resistant polymer materials in recent years [21-23]. The heat release rate (HRR) curves for cellulose and its TOC were shown in Figure 7. The total heat release (THR) curves for cellulose and its TOC were shown in Figure 8. The other MCC data were shown in Table 2.

From Figure 7, the peak heat release rate (pHHR) of TOC was significantly reduced compared to that for pure cellulose. The pHHR of cellulose-COONa was reduced by nearly $90 \%$, when compared with the original cellulose, in which the effect was significant. Simultaneously, the THR of cellulose is $18 \mathrm{KJ} / \mathrm{g}$, and the THR of all TOC is also significantly reduced (Table 2, Figure 8). It is also seen that the amount of combustion residue (Table 2) of the TOC is increased, which is consistent with the results of TG analysis.

Other studies have shown that the presence of metal oxides in polymers can promote the formation of carbon residue $[24,25]$. Therefore, we can infer that, owing to the presence of a large amount of metal ions in TOC, the amount of combustion residue increased, thereby further reducing the THR.

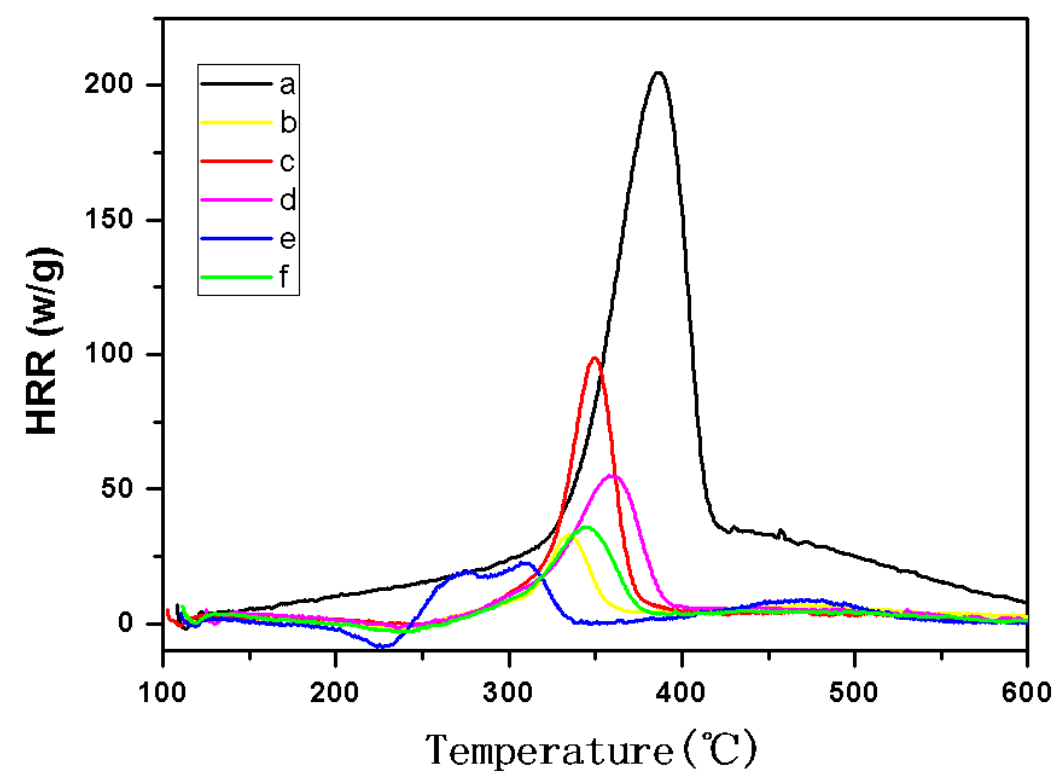

Figure 7. The heart release rate (HRR) curves of cellulose (a), cellulose-COOZn (b), cellulose-COOMg (c), cellulose-COOCa (d), cellulose-COONa (e), and cellulose-COOBa (f) at $1 \mathrm{~K} / \mathrm{s}$ heating rate.

Table 2. Microscale combustion calorimetry (MCC) data of cellulose and TOC.

\begin{tabular}{ccccc}
\hline Samples & pHHR $(\mathbf{w} / \mathbf{g})$ & $\mathbf{T}_{\mathbf{p H H R}}\left({ }^{\circ} \mathbf{C}\right)$ & THR $(\mathbf{K J} / \mathbf{g})$ & Combustion Residue $\left(\mathbf{6 0 0}{ }^{\circ} \mathbf{C}, \mathbf{\%}\right)$ \\
\hline cellulose & 204.7 & 388 & 18.0 & 8.57 \\
cellulose-COOZn & 33.0 & 335 & 2.8 & 35.51 \\
cellulose-COOMg & 98.8 & 350 & 4.7 & 28.55 \\
cellulose-COOCa & 55.0 & 358 & 4.2 & 32.65 \\
cellulose-COONa & 22.7 & 307 & 2.2 & 29.73 \\
cellulose-COOBa & 36.0 & 345 & 2.7 & 35.89 \\
\hline
\end{tabular}

\subsection{LOI Test Analysis}

The LOI test is one of the most commonly used test methods in studying the combustion properties of materials. The larger the value of LOI, the higher the oxygen concentration required for the material to burn, the harder it is to burn the material, and the better its flame-retardant performance. The LOI 
test was conducted to investigate the flame resistance of wood pulp before and after the modification. The corresponding results from the test are summarized in Table 3.

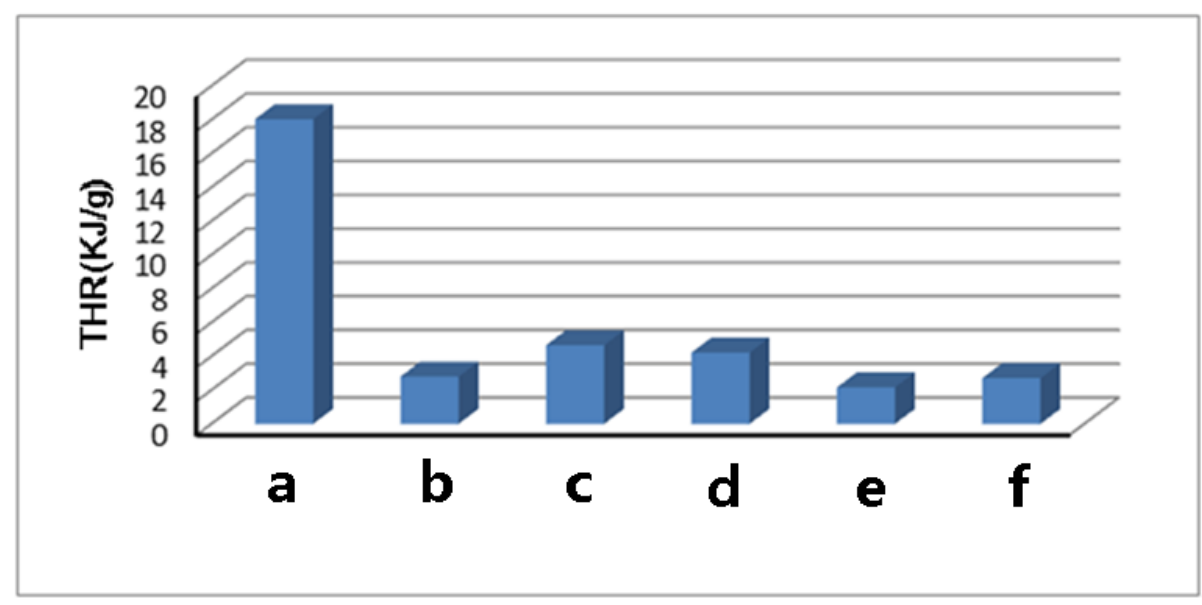

Figure 8. The total heart release (THR) of cellulose (a), cellulose-COOZn (b), cellulose-COOMg (c), cellulose-COOCa (d), cellulose-COONa (e), and cellulose-COOBa (f).

Table 3. The result of LOI of cellulose and TOC.

\begin{tabular}{cc}
\hline Samples & LOI (\%) \\
\hline Cellulose & 19 \\
cellulose-COOZn & 34 \\
cellulose-COOMg & 27 \\
cellulose-COOCa & 25 \\
cellulose-COONa & 27 \\
cellulose-COOBa & 25 \\
\hline
\end{tabular}

The LOI value of the original cellulose is $19 \%$, and the LOI values of all TOCs products are more than $25 \%$, wherein the highest LOI value is that of cellulose-COOZn is $34 \%$. As can be seen from the results, the introduction of carboxyl groups and metal ions by selective oxidation of cellulose, the flame retardancy of the material is improved to some extent.

\section{Materials and Methods}

\subsection{Materials}

Sodium bromide (NaBr) (Guangdong Guanghua Chemical Factory Co., Ltd., China), 2,2,6,6tetramethylpiperidine-1-oxyl radical (TEMPO, 98\%), (J\&K Scientific Ltd, Shanghai, China), and ten mass \% (10 wt\%) sodium hypochlorite $(\mathrm{NaClO})$ solution (Tianjin Dingshengxin Chemical Industry Co., Ltd., China). Other chemicals were analytical grades and were used without prior purification. Cellulose wood pulp (Shandong Sun Paper Industry Joint Stock Co., Ltd., Jining, China) was used as the original cellulose sample.

\subsection{Synthesis of TOC}

The wood pulp $(16 \mathrm{~g})$ was suspended in purified water $(1200 \mathrm{~mL})$ which contained TEMPO $(0.25 \mathrm{~g}$, $0.1 \mathrm{mmol} / \mathrm{g}$ cellulose) and sodium bromide (1.65 g, $1.0 \mathrm{mmol} / \mathrm{g}$ cellulose). Then, a predetermined amount of the $10 \% \mathrm{NaClO}$ solution $(78 \mathrm{~mL}, 5.0-10.0 \mathrm{mmol} / \mathrm{g}$ cellulose) was added to the slurry in dropwise, and the mixture was mechanically stirred at room temperature. Meanwhile the $\mathrm{pH}$ of the mixture system was maintained to be 10.5 until no $\mathrm{NaOH}(0.5 \mathrm{~mol} / \mathrm{L})$ consumption was observed in the reaction process. The oxidation was quenched by adding ethanol (ca.10 mL). The oxidized cellulose was washed thoroughly with purified water and then ethanol by filtration. The wet product was dried 
by lyophilization, followed by vacuum-drying at $50{ }^{\circ} \mathrm{C}$ for about $48 \mathrm{~h}$, and finally weighed to measure the mass recovery ratios.

After being TEMPO-oxidized, the wet product was suspended in an aqueous metal salt solution (1200 mL). The molar concentration of metal salt dissolved in the aqueous cellulose slurry was set to be about three times as much as original wood pulp in the slurry. After stirring the cellulose slurry at room temperature for $6 \mathrm{~h}$, the fibrous cellulose fraction was washed thoroughly with a great deal of deionized water (ca. $4000 \mathrm{~mL}$ ) followed by filtration. Then the filter cake was dried by lyophilization followed by vacuum drying at $50{ }^{\circ} \mathrm{C}$ for $48 \mathrm{~h}$. The following metal salts (magnesium chloride, calcium chloride, zinc chloride, and barium chloride) were used for the ion-exchange treatments. The resulting metal-exchanged TOC was labeled as Cellulose-COOM, where $\mathrm{M}=\mathrm{Na}, \mathrm{Mg}, \mathrm{Ca}, \mathrm{Zn}$, or Ba.

\subsection{Characterization of TOC}

\subsubsection{Fourier Transform Infrared (FT-IR) Spectroscopy}

The structural changes were measured with a FT-IR NICOLET5700 spectrometer (Thermo Nicolet Corporation, Verona, State abbreviation, USA). Samples were studied as KBr pellets ( $1 \%$ in anhydrous $\mathrm{KBr}$ ). Spectra were recorded in the range of from 4000 to $400 \mathrm{~cm}^{-1}$ with a resolution of $2 \mathrm{~cm}^{-1}$, and 20 scans were accumulated. In order to ensure the accuracy of detection, a blank run was made prior to every sample, and the spectrum of the blank was subtracted from the spectrum of the sample.

\subsection{2. ${ }^{13} \mathrm{C}-\mathrm{NMR}$ Spectroscopy}

In order to further analyze the structure of before and after oxidized cellulose, a solid-state ${ }^{13} \mathrm{C}-\mathrm{NMR}$ was used. The spectra were recorded on a Bruker AVANCE_III 300 Superconducting solid spectrometer (Brugg Group, Bruker, Swizterland) with operating frequency of $75.5 \mathrm{MHz}$ and the main magnetic field strength of $7.05 \mathrm{~T}, \mathrm{CP} / \mathrm{TOSS}$ pulse sequence, $75,000 \mathrm{~Hz}$ spectral width, 4096 data points, $5 \mu$ s pulse length, and $5 \mathrm{~s}$ relaxation delay. Scans of up to 1024 were accumulated.

\subsubsection{Thermogravimetry (TG)}

The thermal stabilities of the samples were carried out by recording TG curve on a thermogravimetric analysis equipment (Q500, TA Instrument, Newcastle, USA). Approximately $8 \mathrm{mg}$ of each sample was heated to $900^{\circ} \mathrm{C}$ at a heating rate of $10^{\circ} \mathrm{C} / \mathrm{min}$. All of the measurements were performed under a nitrogen atmosphere with a gas flow of $20 \mathrm{~mL} / \mathrm{min}$ in order to prevent any thermo-oxidative degradation. TG measurements were duplicated in order to assess their reproducibility.

\subsubsection{Microscale Combustion Calorimetry (MCC)}

The samples were pyrolyzed in a microscale combustion calorimetry (Govmark MCC-2, Govmark Organization, Inc.NewYork, USA) to $900{ }^{\circ} \mathrm{C}$ at $1 \mathrm{k} / \mathrm{s}$ under nitrogen atmosphere. The volatiles were swept out continuously by a $\mathrm{N}_{2}$ flow $(80 \mathrm{~mL} / \mathrm{min})$, mixed with a metered flow of oxygen gas $\left(20 \mathrm{~mL} / \mathrm{min}\right.$ ), and completely combusted at $900^{\circ} \mathrm{C}$. Consumption rate of $\mathrm{O}_{2}$ was measured continuously. The heat release results were taken as the average of five measurements for each sample.

\subsubsection{Limiting Oxygen Index (LOI) Test}

The flame retardancy of the prepared samples was tested by the HC-2 type limiting oxygen index meter (Nanjing Jiangning District Analytical Instrument Factory, China) according to GB4545-1997. Each fibrous sample was directly compressed, and the sample size was $100 \mathrm{~mm} \times 37 \mathrm{~mm} \times 2 \mathrm{~mm}$ (ca. $3 \mathrm{~g}$ ).

\section{Conclusions}

The C6 primary hydroxyl group of cellulose can be electively oxidized to a carboxyl group by TEMPO-mediated oxidation system, and further various metal ions are introduced in the polymers by 
ion-exchange method. The thermal stabilities of the prepared TOC were significantly reduced after modification because of the increase of carboxyl groups and the addition of metal ions during the oxidation and ion- exchange due to the decrease in crystallinity of TOC. However, the MMLR of TOC was significantly lower than that of cellulose. This can be speculated in that various TOC which contain more carboxyl groups will release a large amount of nonflammable gas $\left(\mathrm{CO}_{2}\right)$ and dilute the flammable gas during degradation at a lower temperature. Additionally, it is more easily to generate combustion residue at a relatively low temperature. Furthermore, it can be seen from the data of MCC that the HRR and THR of TOC were significantly reduced. The LOI values of all TOCs products were much higher than $25 \%$, which indicated that the flame retardancy of the modified cellulose was improved.

Author Contributions: The authors contributed as follows Y.X., C.G., Z.Z., and Z.X. in conceptualization, visualization and methodology and synthesis, C.G., Y.X., P.X, Z.X. and Z.Z. in writing-original draft preparation, P.X., Y.X. and C.G. in writing-review and editing; supervision, project administration, and Y.X. and Z.Z. in funding acquisition.

Funding: The authors are grateful for the support of the National Key R\&D Program of China (NO. 2017YFB0309005), National Natural Science Foundation of China (Grant No. 51303089 and 41807499), Key R\&D Program of Shandong Province (NO.2017GNC11113), Research Project of Innovative Teaching Laboratory of Qingdao University and Taishan Scholar Program of Shandong Province.

Acknowledgments: The authors acknowledge Peng Liu from Institute of Chemistry Chinese Academy of Sciences for the MCC test of TOC.

Conflicts of Interest: The authors declare no conflict of interest.

\section{References}

1. Ifuku, S.; Kadla, J.F. Preparation of a thermosensitive highly regioselective cellulose/ $N$-isopropylacrylamide copolymer through atom transfer radical polymerization. Biomacromolecules 2008, 9, 3308-3313. [CrossRef] [PubMed]

2. Lessan, F.; Montazer, M.; Moghadam, M.B. A novel durable flame-retardant cotton fabric using sodium hypophosphite, nano $\mathrm{TiO}_{2}$ and maleic acid. ThermochimActa 2011, 520, 48-54. [CrossRef]

3. Shariatinia, Z.; Javeri, N.; Shekarriz, S. Flame retardant cotton fibers produced using novel synthesized halogen- free phosphoramide nanoparticles. CarbohydrPolym 2015, 118, 183-198. [CrossRef]

4. Xie, K.L.; Gao, A.Q.; Zhang, Y.S. Flame retardant finishing of cotton fabric based on synergistic compounds containing boron and nitrogen. CarbohydrPolym 2013, 98, 706-710. [CrossRef]

5. Alongi, J.; Carletto, R.A.; Bosco, F.; Carosio, F.; Di Blasio, A.; Cuttica, F.; Antonucci, V.; Giordano, M.; Malucelli, G. Caseins and hydrophobins as novel green flame retardants for cotton fabrics. PolymDegrad Stab 2014, 99, 111-117. [CrossRef]

6. Salmeia, K.A.; Gaan, S.; Malucelli, G. Recent advances for flame retardancy of textiles based on phosphorus chemistry. Polymers 2016, 8, 319. [CrossRef] [PubMed]

7. Feng, Y.J.; Zhou, Y.; Li, D.K.; He, S.; Zhang, F.X.; Zhang, G.X. A plant-based reactive ammonium phytate for use as a flame-retardant for cotton fabric. CarbohydrPolym 2017, 175, 636-644. [CrossRef] [PubMed]

8. Ghanadpour, M.; Carosio, F.; Larsson, P.T.; Wågberg, L. Phosphorylated Cellulose Nanofibrils: A Renewable Nanomaterial for the Preparation of Intrinsically Flame-Retardant Materials. Biomacromolecules 2015, 16, 3399-3410. [CrossRef]

9. Kong, Q.S.; Wang, B.B.; Ji, Q.; Xia, Y.Z.; Guo, Z.X.; Yu, J. Thermal degradation and flame retardancy of calcium alginate fibers. Chinese J. Polym. Sci. 2009, 27, 807-812. [CrossRef]

10. Zhang, J.J.; Ji, Q.; Shen, X.H.; Xia, Y.Z.; Tan, L.W.; Kong, Q.S. Pyrolysis products and thermal degradation mechanism of intrinsically flame-retardant calcium alginate fibre. Polym. Degrad. Stabil. 2011, 96, 936-942. [CrossRef]

11. Shibata, I.; Isoga, A. Depolymerization of cellouronic acid during TEMPO-mediated oxidation. Cellulose 2003, 10, 151-158. [CrossRef]

12. Saito, T.; Isogai, A. TEMPO-mediated oxidation of native cellulose. The effect of oxidation conditions on chemical and crystal structures of the waterinsoluble fractions. Biomacromolecules 2004, 5, 1983-1989. [CrossRef] 
13. Isogai, A.; Kato, Y. Preparation of polyuronic acid from cellulose by TEMPO-mediated oxidation. Cellulose 1998, 5, 153-164. [CrossRef]

14. Saito, T.; Nishiyama, Y.; Putaux, J.L.; Vignon, M.; Isogai, A. Homogeneous Suspensions of Individualized Microfibrils from TEMPO-Catalyzed Oxidation of Native Cellulose. Biomacromolecules 2006, 7, 1687-1691. [CrossRef] [PubMed]

15. Ifuku, S.; Tsuji, M.; Morimoto, M.; Saimoto, H.; Yano, H. Synthesis of silver nanoparticles template by TEMPO-mediated oxidized bacterial cellulose nanofibers. Biomacromolecules 2009, 10, 2714-2717. [CrossRef] [PubMed]

16. Keshk; Sherif, M.A.S. Homogenous reactions of cellulose from different natural sources. Carbohyd. Polym. 2008, 74, 942-945. [CrossRef]

17. Saito, T.; Shibata, I.; Isogai, A.; Suguri, N.; Sumikwa, N. Distribution of carboxylate groups introduced into cotton linters by the TEMPO-mediated oxidation. Carbohyd. Polym. 2005, 61, 414-419. [CrossRef]

18. Vieira, M.C.; Heinze, T.; Antonio-Cruz, R.; Mendoza-Martinez, A.M. Cellulose derivatives from cellulosic material isolated from Agave lechuguilla and fourcroydes. Cellulose 2002, 9, 203-212. [CrossRef]

19. Zhang, H.; Westmoreland, P.R.; Farris, F.J.; Coughlin, E.B.; Plichta, A.; Brzozowski, Z.K. Thermal decomposition and flammability of fire-resistant, UV/visible-sensitive polyarylates, copolymers and blends. Polymer 2002, 43, 5463-5472. [CrossRef]

20. Tripathy, A.R.; Farris, F.; Macknight, W.J. Novel fire resistant matrixes for composites from cyclic poly (butylenes terephthalate). Polym. Eng. Sci. 2007, 47, 1536-1543. [CrossRef]

21. Lyon, R.E.; Walters, R.N. Pyrolysis combustion flow calorimetry. J. Anal. Appl. Pyrol. 2004, 71, 27-46. [CrossRef]

22. Lyon, R.E.; Walters, R.N.; Stoliarov, S.I. Screening flame retardants for plastics using microscale combustion calorimetry. Polym. Eng. Sci. 2007, 47, 1501-1510. [CrossRef]

23. Tai, Q.L.; Kan, Y.C.; Chen, L.J.; Xing, W.Y.; Hu, Y.; Song, L. Morphologies and thermal properties of flame-retardant polystyrene/ a -zirconium phosphate nanocomposites. React. Funct. Polym. 2010, 70, 340-345. [CrossRef]

24. Laoutid, F.; Ferry, L.; Lopez-Cuesta, J.M.; Crespy, A. Flame-retardant action of red phosphorus/magnesium oxide and red phosphorus/iron oxide compositions in recycled PET. Fire Mater 2006, 30, 343-358. [CrossRef]

25. Levchik, G.F.; Vorobyova, S.A.; Gorbarenko, V.V.; Levchik, S.V.; Weil, E.D. Some mechanistic aspects of the fire retardant action of red phosphorus in aliphatic nylons. J. Fire Sci. 2000, 18, 172-182. [CrossRef]

Sample Availability: Samples of the TOC are available from the authors.

(C) 2019 by the authors. Licensee MDPI, Basel, Switzerland. This article is an open access article distributed under the terms and conditions of the Creative Commons Attribution (CC BY) license (http://creativecommons.org/licenses/by/4.0/). 